Research Article

\title{
On the Lattice Properties of Almost L-Weakly and Almost M- Weakly Compact Operators
}

\author{
Barış Akay $\mathbb{D}^{1}$ and Ömer Gök (iD) ${ }^{2}$ \\ ${ }^{1}$ Department of Mathematics, Science Faculty, Istanbul University, Istanbul 34134, Turkey \\ ${ }^{2}$ Department of Mathematics, Faculty of Arts and Sciences, Yildiz Technical University, Istanbul 34220, Turkey \\ Correspondence should be addressed to Barış Akay; baris.akay@istanbul.edu.tr
}

Received 13 April 2021; Revised 3 May 2021; Accepted 7 May 2021; Published 20 May 2021

Academic Editor: Calogero Vetro

Copyright (C) 2021 Barış Akay and Ömer Gök. This is an open access article distributed under the Creative Commons Attribution License, which permits unrestricted use, distribution, and reproduction in any medium, provided the original work is properly cited.

\begin{abstract}
We establish the domination property and some lattice approximation properties for almost L-weakly and almost M-weakly compact operators. Then, we consider the linear span of positive almost L-weakly (resp., almost M-weakly) compact operators and give results about when they form a Banach lattice and have an order continuous norm.
\end{abstract}

\section{Introduction and Notation}

In this article, we denote real Banach spaces by $X$ and $Y$ and real Banach lattices by $E$ and $F$. The closed unit ball and the norm dual of $X$ are denoted by $B_{X}$ and $X^{\prime}$, respectively. $E_{+}$ denotes the positive cone of $E$, i.e., $E_{+}=\{x \in E: x \geq 0\}$. Let $x \in E$. The positive part, the negative part, and the modulus of $x$ are given by $x^{+}=x \vee 0, x^{-}=(-x) \vee 0$, and $|x|=(-x) \vee x$, respectively. For all $x, y \in E$ with $x \leq y$, the order interval between $x$ and $y$ is denoted by $[x, y]=\{z \in E: x \leq z \leq y\}$. We write $\operatorname{Sol}(A)$ for the solid hull of a set $A \subseteq E$. By an operator $T: X \longrightarrow Y$, we mean a bounded linear mapping. The space of all operators from $X$ into $Y$ is denoted by $\mathscr{L}(X, Y)$ . If $T: X \longrightarrow Y$ is an operator, its adjoint $T^{\prime}: Y^{\prime} \longrightarrow X^{\prime}$ is defined by $\left(T^{\prime} f\right)(x)=f(T x)$ for each $f \in Y^{\prime}$ and for each $x$ $\in X$. The space of all regular operators from $E$ into $F$ is denoted by $\mathscr{L}^{r}(E, F)$. If $T: E \longrightarrow F$ is an operator with modulus, then the regular norm of $T$ is given by $\|T\|_{r}=\||T|\|$. For any unexplained notion and terminology, we refer to $[1,2]$.

Recently, the class of compact and related operators was studied extensively (for instance in [3, 4]). In [3], some identities and estimates for the Hausdorff measures of noncompactness of some operators on the fractional sets of sequences of fractional orders were established, and some classes of compact operators on the fractional sets of sequences were characterized. Also, necessary and sufficient conditions for the class of compact matrix operators from the fractional sets of sequences into the set of bounded sequences were given. In [4], power bounded $m$-isometric Banach space operator was shown to be polaroid, and the polaroid property for $n$-quasi left $m$-invertible operators was proved. In approximation theory, many authors studied some estimates on the positive linear operators with an emphasis on the Kantorovich operators, DurrmeyerBernstein operators, and exponential type operators [5-7]. These types of operators have nice and interesting convergence properties. The approximation process by the sequence of positive linear operators for integrable or continuous functions was presented [5-7]. The common properties of these studies and the present work are positivity of linear operators, uniform convergence of sequences, and Banach lattices (e.g., $L_{1}[0,1]$ and $\left.\mathrm{C}[0,1]\right)$.

The class of compact (resp., weakly compact) operators does not satisfy the domination property $[1,2]$. In other words, if two positive operators $S, T: E \longrightarrow F$ between Banach lattices satisfy $0 \leq S \leq T$ and $T$ is compact (resp., weakly compact), then $S$ is not necessarily compact (resp., weakly compact). Also, a compact operator (resp., weakly 
compact operator) between Banach lattices need not possess a modulus ([1], p. 277). In [8], Meyer-Nieberg introduced the classes of L-weakly and M-weakly compact operators to overcome some difficulties in studying compact and weakly compact operators. Recall that an operator $T: X \longrightarrow F$ is called L-weakly compact if $T\left(B_{X}\right)$ is an L-weakly compact set, i.e., every disjoint sequence in the solid hull of $T\left(B_{X}\right)$ converges to zero in norm. An operator $T: E \longrightarrow Y$ is called M-weakly compact if for each norm bounded disjoint sequence $\left(x_{n}\right)$ in $E$, we have $\left\|T x_{n}\right\| \longrightarrow 0$. Note that $T: E \longrightarrow F$ is L-weakly compact (resp., M-weakly compact) if and only if its adjoint $T^{\prime}$ is M-weakly compact (resp., L-weakly compact) ([2], Proposition 3.6.11). In contrast to compact and weakly compact operators, the domination property holds for L-weakly (resp., M-weakly) compact operators ([9], Proposition 2.1). However, an L-weakly (resp., M-weakly) compact operator need not have a modulus ([10], Theorem 2.2). In order to study these operator classes as vector lattices, Bayram and Wickstead worked in the linear span of positive L-weakly (resp., M-weakly) compact operators between Banach lattices [9]. For more details about these classes of operators, we refer to $[1,2,8-10]$.

The class of almost L-weakly (resp., almost M-weakly) compact operators was introduced in [11] as a generalization of that of L-weakly (resp., M-weakly) compact operators. Recall that an operator $T: X \longrightarrow F$ is called almost $\mathrm{L}$ weakly compact if $T$ maps relatively weakly compact subsets of $X$ onto L-weakly compact subsets of $F$, and an operator $T: E \longrightarrow Y$ is called almost $\mathrm{M}$-weakly compact if for each disjoint sequence $\left(x_{n}\right)$ in $B_{E}$ and for each weakly convergent sequence $\left(f_{n}\right)$ in $Y^{\prime}$, we have $f_{n}\left(T x_{n}\right) \longrightarrow 0$. Every L-weakly (resp., M-weakly) compact operator is almost L-weakly (resp., almost M-weakly) compact, but the converse is not true in general [11]. For example, the identity operator $I$ $: \ell_{1} \longrightarrow \ell_{1}$ is almost L-weakly compact but not L-weakly compact, and the identity operator $I: \ell_{\infty} \longrightarrow \ell_{\infty}$ is almost M-weakly compact but not M-weakly compact ([11], p. 1435). Note that an operator $T: E \longrightarrow Y$ is almost $M-$ weakly compact if and only if its adjoint $T^{\prime}$ is almost Lweakly compact, and an operator $T: X \longrightarrow F$ is almost $\mathrm{L}$ weakly compact whenever its adjoint $T^{\prime}$ is almost $M-$ weakly compact ([11], Theorem 2.5). The relationship between almost L-weakly (resp., almost M-weakly) compact operators and other classes of operators (e.g., compact operators, weakly compact operators, L-weakly, and M-weakly compact operators) was studied in the literature [11-13].

In the sequel, we will use the following notations:

$W_{L}(E, F)$ is the space of L-weakly compact operators from $E$ into $F$

$W_{M}(E, F)$ is the space of M-weakly compact operators from $E$ into $F$

$A W_{L}(E, F)$ is the space of almost L-weakly compact operators from $E$ into $F$

$A W_{M}(E, F)$ is the space of almost M-weakly compact operators from $E$ into $F$

$$
\begin{aligned}
& W_{L}^{r}(E, F)=\left\{T_{1}-T_{2}: T_{1}, T_{2} \in W_{L}(E, F)_{+}\right\} \\
& W_{M}^{r}(E, F)=\left\{T_{1}-T_{2}: T_{1}, T_{2} \in W_{M}(E, F)_{+}\right\} \\
& A W_{L}^{r}(E, F)=\left\{T_{1}-T_{2}: T_{1}, T_{2} \in A W_{L}(E, F)_{+}\right\} \\
& A W_{M}^{r}(E, F)=\left\{T_{1}-T_{2}: T_{1}, T_{2} \in A W_{M}(E, F)_{+}\right\}
\end{aligned}
$$

In this paper, our aim is to study the vector lattice properties of almost L-weakly and almost M-weakly compact operators. First, we will show that both classes of operators satisfy the domination property. To establish this, we will make use of the class of almost Dunford-Pettis operators, which was introduced by Sanchez [14]. Next, we will present some lattice approximation properties for almost L-weakly and almost $\mathrm{M}$-weakly compact operators. Then, we will consider the spaces $A W_{L}^{r}(E, F)$ and $A W_{M}^{r}(E, F)$ and investigate whether these spaces are Banach lattices with the regular norm. Here, we will make use of the domination property and obtain analogous results to that of [9]. Finally, we will present some necessary and sufficient conditions for $A W_{L}^{r}($ $E, F)$ and $A W_{M}^{r}(E, F)$ to have an order continuous regular norm.

Let us recall some definitions and well-known facts. A Banach lattice $E$ is said to have an order continuous norm if for each net $\left(x_{\alpha}\right)$ in $E$ with $x_{\alpha} \downarrow 0$, we have $\left\|x_{\alpha}\right\| \longrightarrow 0$. Here, the notation $x_{\alpha} \downarrow 0$ means that $\left(x_{\alpha}\right)$ is decreasing, its infimum exists, and inf $\left(x_{\alpha}\right)=0$. By $E^{a}$, we denote the maximal (order) ideal of $E$ on which the induced norm is order continuous. Note that $E^{a}$ is closed, and every L-weakly compact subset of $E$ is contained in $E^{a}$ ([2], p. 212). Let $\rho$ be a lattice seminorm on $E$. A subset $A$ of $E$ is called approximately order bounded with respect to $\rho$ if for every $\varepsilon>0$ there exists $u \in$ $E_{+}$such that $A \subseteq[-u, u]+\varepsilon B_{\rho}$, where $B_{\rho}=\{x \in E: \rho(x) \leq 1\}$ ([2], p. 73). We have $A \subseteq[-u, u]+\varepsilon B_{\rho}$ if and only if $\rho($ $\left.(|x|-u)^{+}\right) \leq \varepsilon$ for all $x \in A([2]$, p. 73$)$. An operator $T: X$ $\longrightarrow F$ is called semicompact if for each $\varepsilon>0$ there exists $u$ $\in F_{+}$such that $T\left(B_{X}\right) \subseteq[-u, u]+\varepsilon B_{F}$, i.e., $T\left(B_{X}\right)$ is an approximately order bounded set in $F$. Let $T: E \longrightarrow Y$ be an operator. The lattice seminorm $q_{T}$ on $E$ is given by $q_{T}(x$ )$=\sup \{\|T(y)\|:|y| \leq|x|\}$ ([2], p. 192). From [14, 15], an operator $T: E \longrightarrow Y$ is called almost Dunford-Pettis if for every weakly null sequence $\left(x_{n}\right)$ consisting of pairwise disjoint elements in $E$, we have $\left\|T x_{n}\right\| \longrightarrow 0$.

\section{Main Results}

2.1. Domination Property. In the following result, we show that the class of almost L-weakly compact operators satisfies the domination property.

Theorem 1. Let $E$ and $F$ be Banach lattices and $S, T: E \longrightarrow F$ be positive operators satisfying $0 \leq S \leq T$. If $T$ is almost $L$ weakly compact, then $S$ is also almost L-weakly compact.

Proof. Let $T: E \longrightarrow F$ be a positive almost L-weakly compact operator. It follows from Proposition 2.3 of [11] that $T$ is almost Dunford-Pettis. It is known that the class of almost Dunford-Pettis operators satisfies the domination property ([16], Corollary 2.3). Hence, the operator $S$ is almost Dunford-Pettis. Now, let $W \subseteq E$ be a relatively weakly compact subset and $\varepsilon>0$. Since $S$ is almost Dunford-Pettis, the set $W$ is approximately order bounded with respect to the lattice seminorm $q_{S}$ ([16], Proposition 2.1). So, there exists 
some $u \in E_{+}$such that

$$
\left\|S(|x|-u)^{+}\right\| \leq q_{S}\left((|x|-u)^{+}\right) \leq \varepsilon,
$$

for all $x \in W$. On the other hand, since $T$ is almost L-weakly compact, we have $T(E) \subseteq F^{a}$ ([12], Proposition 1). By using the fact that $0 \leq S \leq T$ and $F^{a}$ is a solid set, it is easy to see that $S(E) \subseteq F^{a}$. Put $z=S u \in F_{+}^{a}$. Then, for all $x \in W$, we have

$$
\begin{aligned}
(|S x|-z)^{+} & =(|S x|-S u)^{+} \leq(S|x|-S u)^{+} \\
& \leq(S(|x|-u))^{+} \leq S(|x|-u)^{+} .
\end{aligned}
$$

By using (1) and (2), we obtain $\left\|(|S x|-z)^{+}\right\| \leq \varepsilon$ for all $x \in W$. From the identity $|S x|=|S x| \wedge z+(|S x|-z)^{+}$, we see that $S(W) \subseteq[-z, z]+\varepsilon B_{F}$. Since $z \in F_{+}^{a}$, the set $S(W)$ is approximately order bounded in $F^{a}$. Therefore, $S(W)$ is an L-weakly compact set ([2], Proposition 3.6.2). As a result, $S$ is an almost L-weakly compact operator.

The domination property for almost M-weakly compact operators follows easily from Theorem 1.

Theorem 2. Let $E$ and $F$ be Banach lattices and $S, T: E \longrightarrow F$ be positive operators satisfying $0 \leq S \leq T$. If $T$ is almost $M$ weakly compact, then $S$ is also almost M-weakly compact.

Proof. Clearly, we have $0 \leq S^{\prime} \leq T^{\prime}$. Since $T$ is almost Mweakly compact, its adjoint $T^{\prime}$ is almost L-weakly compact ([11], Theorem 2.5). Theorem 1 yields that the operator $S^{\prime}$ is also almost L-weakly compact. Using Theorem 2.5 of [11] again, we conclude that $S$ is almost M-weakly compact.

2.2. Lattice Approximation Properties. The class of almost Lweakly compact operators satisfies the following lattice approximation property.

Proposition 3. Let $T: X \longrightarrow F$ be an almost L-weakly compact operator from a Banach space $X$ into a Banach lattice $F$ . If $W$ is a relatively weakly compact subset of $X$, then for each $\varepsilon>0$, there exists $u_{\varepsilon} \in F_{+}$lying in the ideal generated by $T(W)$ such that

$$
\left\|\left(|T x|-u_{\varepsilon}\right)^{+}\right\|<\varepsilon,
$$

for all $x \in W$.

Proof. Let $W \subseteq X$ be a relatively weakly compact set. Consider the solid hull of $T(W)$ and call it $A$. Clearly, the set $A$ is bounded and solid. Since $T$ is almost L-weakly compact, each disjoint sequence in $A$ converges to zero in norm. Define the identity operator $I: F \longrightarrow F$ and let $p(x)=\|x\|$. If $\left(x_{n}\right)$ is an arbitrary disjoint sequence in $A$, then $p\left(I\left(x_{n}\right)\right)=\left\|x_{n}\right\| \longrightarrow 0$. Let $\varepsilon>0$. It follows from Theorem 4.36 of [1] that there exists some $u \in E_{+}$lying in the ideal generated by $A$ such that

$$
\left\|I(|y|-u)^{+}\right\|<\varepsilon
$$

for all $y \in A$. Since $T(W) \subseteq A$, we have

$$
\left\|(|T x|-u)^{+}\right\|<\varepsilon,
$$

for all $x \in W$. As the ideal generated by $T(W)$ is equal to the ideal generated by $A$, we obtain the desired result.

For the class of almost M-weakly compact operators, we first give a characterization and obtain from that a lattice approximation property.

Proposition 4. A positive operator $T: E \longrightarrow F$ between Banach lattices is almost M-weakly compact if and only if given any relatively weakly compact set $V \subseteq F^{\prime}$ and a disjoint sequence $\left(x_{n}\right)$ in $B_{E}$, the sequence $\left(T x_{n}\right)$ converges uniformly to zero on the solid hull of $V$.

Proof. For the forward implication, we give a similar proof to that of ([1], Theorem 5.100). Assume that $T$ is almost Mweakly compact. Let $V \subseteq F^{\prime}$ be a relatively weakly compact set and $\left(x_{n}\right) \subseteq E_{+}$be a disjoint sequence in $B_{E}$. Fix $\varepsilon>0$. First, we claim that there exist some $g \in\left(F^{\prime}\right)_{+}$and $k \in \mathbb{N}^{+}$such that

$$
(|f|-g)^{+}\left(T x_{n}\right)<\varepsilon,
$$

for all $f \in V$ and for all $n>k$.

To see this, assume on the contrary that (6) is false. So, for each $g \in\left(F^{\prime}\right)_{+}$and for each $k \in \mathbb{N}^{+}$, there exist $f \in V$ and $m$ $>k$ such that $(|f|-g)^{+}\left(T x_{m}\right) \geq \varepsilon$. By induction, we can see that there exist a sequence $\left(f_{n}\right) \subseteq V$ and a subsequence $\left(z_{n}\right)$ of $\left(x_{n}\right)$ such that

$$
\left(\left|f_{n+1}\right|-4^{n} \sum_{i=1}^{n} \otimes\left|f_{i}\right|\right)^{+}\left(T z_{n}\right) \geq \varepsilon,
$$

for all $n$. Put $f=\sum_{n=1}^{\infty} \bigotimes 2^{-n}\left|f_{n}\right|$ and $h_{n}=$ $\left(\left|f_{n+1}\right|-4^{n} \sum_{i=1}^{n} \bigotimes\left|f_{i}\right|\right)^{+}$. Clearly, we have $h_{n}\left(T z_{n}\right) \geq \varepsilon$ for all $n$. We define

$$
g_{n}=\left(\left|f_{n+1}\right|-4^{n} \sum_{i=1}^{n} \otimes\left|f_{i}\right|-2^{-n} f\right)^{+},
$$

and note that $\left(g_{n}\right)$ is a disjoint sequence in the solid hull of $V$ ([1], Lemma 4.35). From Theorem 4.34 of [1], we see that $g_{n} \longrightarrow{ }^{w} 0$ in $F^{\prime}$. Moreover, $\left(z_{n}\right)$ is a disjoint sequence with positive terms in $B_{E}$. As $T$ is almost M-weakly compact, we have $g_{n}\left(T z_{n}\right) \longrightarrow 0$. Now, define the constant sequence $i_{n}$ $=f$ in $F^{\prime}$. Clearly, $\left(i_{n}\right)$ is weakly convergent. Since $T$ is almost M-weakly compact, we obtain $i_{n}\left(T z_{n}\right)=f\left(T z_{n}\right) \longrightarrow$ 0 . From the inequality $0 \leq h_{n} \leq g_{n}+2^{-n} f$, we have

$$
0<\varepsilon \leq h_{n}\left(T z_{n}\right) \leq g_{n}\left(T z_{n}\right)+2^{-n} f\left(T z_{n}\right) \longrightarrow 0,
$$

a contradiction. Thus, (6) is true. We pick $g \in\left(F^{\prime}\right)_{+}$and $k$ $\in \mathbb{N}^{+}$such that (6) holds. Define the constant sequence $j_{n}$ 
$=g$ in $F^{\prime}$. As $T$ is almost M-weakly compact and $\left(j_{n}\right)$ is weakly convergent, $j_{n}\left(T x_{n}\right)=g\left(T x_{n}\right) \longrightarrow 0$. So there is some $m>k$ such that $g\left(T x_{n}\right)<\varepsilon$ for all $n \geq m$. Let $h \in \operatorname{Sol}(V)$ be arbitrary. Then, there is some $f \in V$ with $|h| \leq|f|$. We have

$\left|h\left(T x_{n}\right)\right| \leq|h|\left(T x_{n}\right) \leq|f|\left(T x_{n}\right) \leq(|f|-g)^{+}\left(T x_{n}\right)+g\left(T x_{n}\right) \leq \varepsilon+\varepsilon=2 \varepsilon$,

for all $n \geq m$. This proves that $\left(T x_{n}\right)$ converges uniformly to zero on the solid hull of $V$.

For the converse, let $\left(x_{n}\right)$ be a disjoint sequence in $B_{E}$ and $\left(f_{n}\right)$ be a weakly convergent sequence in $F^{\prime}$. Put $V=\left\{f_{n}\right.$ $: n \in \mathbb{N}\}$. Then, $V$ is a relatively weakly compact subset of $F^{\prime}$. By assumption, the sequence $\left(T x_{n}\right)$ converges uniformly to zero on the solid hull of $V$; that is, $\sup _{f \in \operatorname{Sol}(V)}\left|f\left(T x_{n}\right)\right| \longrightarrow 0$.

For all $n$, we have

$$
\left|f_{n}\left(T x_{n}\right)\right| \leq \sup _{f \in V}\left|f\left(T x_{n}\right)\right| \leq \sup _{f \in \operatorname{Sol}(V)}\left|f\left(T x_{n}\right)\right|
$$

So $\left|f_{n}\left(T x_{n}\right)\right| \longrightarrow 0$, and hence, $T$ is almost M-weakly compact.

Corollary 5. Let $T: E \longrightarrow F$ be a positive almost $M$-weakly compact operator between Banach lattices and $V \subseteq F^{\prime}$ be a relatively weakly compact set. Then, for each $\varepsilon>0$, there exists $u_{\varepsilon} \in E_{+}$satisfying

$$
|f|\left(T\left(|x|-u_{\varepsilon}\right)^{+}\right)<\varepsilon,
$$

for all $x \in B_{E}$ and for all $f \in V$.

Proof. Define the seminorm $\rho$ on $E$ by $\rho(x)=\sup \{|f|(|x|)$ : $f \in V\}$ ([1], Theorem 5.100 (2)). Then, $\rho$ is continuous on $E$. From Proposition 4 , we have $\rho\left(T x_{n}\right) \longrightarrow 0$ for each disjoint sequence $\left(x_{n}\right)$ in $B_{E}$. Since $B_{E}$ is a solid set, Theorem 4.36 of [1] yields that for each $\varepsilon>0$ there exists $u_{\varepsilon} \in E_{+}$satisfying

$$
|f|\left(T\left(|x|-u_{\varepsilon}\right)^{+}\right)<\varepsilon,
$$

for all $f \in V$ and for all $x \in B_{E}$.

2.3. The Spaces $A W_{L}^{r}(E, F)$ and $A W_{M}^{r}(E, F)$. In this section, we study the Banach lattice properties of $A W_{L}^{r}(E, F)$ and $A$ $W_{M}^{r}(E, F)$. In ([9], Theorem 2.2), Bayram and Wickstead proved that $W_{L}^{r}(E, F)$ is always a Dedekind complete Banach lattice with the regular norm. In a similar way, we show that the same is true for $A W_{L}^{r}(E, F)$.

Theorem 6. Let $E$ and $F$ be Banach lattices. Then, $A W_{L}^{r}(E, F)$ , equipped with the regular norm, is a Dedekind complete Banach lattice.

Proof. Let $T \in A W_{L}^{r}(E, F)$. Then, $T=T_{1}-T_{2}$, where $T_{1}, T_{2}$ $\in A W_{L}^{r}(E, F)_{+}$. Put $U=T_{1}+T_{2}$. Clearly, $\pm T \leq U$. Since $T($ $E) \subseteq F^{a}, U(E) \subseteq F^{a}$, and $F^{a}$ is Dedekind complete, it follows that $T$ has a modulus in $\mathscr{L}^{r}\left(E, F^{a}\right)$ and hence in $\mathscr{L}^{r}(E, F)$. As $|T| \leq U$, Theorem 1 yields that $|T| \in A W_{L}^{r}(E, F)$. Thus, $A W_{L}^{r}(E, F)$ is a vector lattice. As $F^{a}$ is Dedekind complete, $A W_{L}^{r}(E, F)$ is also Dedekind complete. Now, we show that $A W_{L}^{r}(E, F)$ is closed in $\mathscr{L}^{r}(E, F)$ with respect to the regular norm. To this end, assume that $T$ is in the closure of $A W_{L}^{r}($ $E, F)$. Then, there exists a sequence $\left(T_{n}\right)$ in $A W_{L}^{r}(E, F)$ such that $\left\|T_{n}-T\right\|_{r} \longrightarrow 0$. Since $T_{n}$ has a modulus for each $n$, Theorem 2.1 of [17] implies that $T$ has a modulus in $\mathscr{L}^{r}(E$, $F)$ and that $\left\|\left|T_{n}\right|-|T|\right\|_{r} \longrightarrow 0$. Therefore, ||$\left|T_{n}\right|-|T| \|$ $\longrightarrow 0$. Since $\left|T_{n}\right| \in A W_{L}(E, F)$ for all $n$ and $A W_{L}(E, F)$ is closed in $\mathscr{L}(E, F)$ ([11], Proposition $2.1(1))$, we have $|T| \epsilon$ $A W_{L}(E, F)$. This shows that $T^{+}$and $T^{-}$are also in $A W_{L}(E$, $F$ ) (by Theorem 1), and so is $T$. As a result, $A W_{L}^{r}(E, F)$ is a Dedekind complete Banach lattice.

Assuming that $F$ is Dedekind complete, we have the following result for almost $\mathrm{M}$-weakly compact operators. The proof is similar to the proof of the above theorem with the corresponding results for almost M-weakly compact operators (Theorem 2 and Proposition 2.1 (2) of [11]).

Theorem 7. Let $E$ and $F$ be Banach lattices with F Dedekind complete. Then, $A W_{M}^{r}(E, F)$, equipped with the regular norm, is a Dedekind complete Banach lattice.

Now, we investigate conditions under which $A W_{L}^{r}(E, F)$ and $A W_{M}^{r}(E, F)$ have an order continuous norm, respectively. For $A W_{L}^{r}(E, F)$, we have exactly the same necessary and sufficient condition with ([9], Theorem 3.1).

Theorem 8. Let $E$ and $F$ be Banach lattices with $F^{a} \neq\{0\}$. Then, the regular norm on $A W_{L}^{r}(E, F)$ is order continuous if and only if $E^{\prime}$ has an order continuous norm.

Proof. Assume that $E^{\prime}$ has an order continuous norm. By Theorem 1, for all $T \in A W_{L}^{r}(E, F)_{+}$, the order intervals $[0, T$ ] are the same in $A W_{L}^{r}(E, F)$ and in $\mathscr{L}^{r}(E, F)$. Let $T \in A W_{L}^{r}$ $(E, F)_{+}$. Since $E^{\prime}$ has an order continuous norm, $T$ is $M-$ weakly compact ([12], Theorem 4). As $T(E) \subseteq F^{a}$ and the norm of $F^{a}$ is order continuous, we conclude that the norm on $[0, T]$ is order continuous ([2], Proposition 3.6.19). Conversely, suppose that the norm on $A W_{L}^{r}(E, F)$ is order continuous. Fix $0 \neq y \in F_{+}^{a}$ and let $\left(f_{\alpha}\right)$ be a net in $E^{\prime}$ such that $f_{\alpha} \downarrow 0$. Define $T_{\alpha}: E \longrightarrow F$ by $T_{\alpha}(x)=f_{\alpha}(x) y$. Then, $T_{\alpha} \downarrow 0$, and hence, $\left\|T_{\alpha}\right\| \longrightarrow 0$ by assumption. So $\left\|T_{\alpha}\right\|=\left\|f_{\alpha}\right\|\|y\|$ $\longrightarrow 0$. This shows that the norm of $E^{\prime}$ is order continuous.

We now turn our attention to $A W_{M}^{r}(E, F)$.

Proposition 9. Let $E$ and $F$ be Banach lattices with $\left(E^{\prime}\right)^{a} \neq$ $\{0\}$. If the regular norm on $A W_{M}^{r}(E, F)$ is order continuous, then $F$ has an order continuous norm.

Proof. Let $\left(y_{\alpha}\right)$ be a net in $F$ such that $y_{\alpha} \downarrow 0$. Fix $0 \neq f \in\left(E^{\prime}\right)^{a}$ and define $T_{\alpha}: E \longrightarrow F$ by $T_{\alpha}(x)=f(x) y_{\alpha}$. It is easily seen that each $T_{\alpha}$ is M-weakly compact (hence almost M-weakly 
compact). Then, for each $x \in E_{+}, T_{\alpha}(x) \downarrow 0$. Thus, $T_{\alpha} \downarrow 0$. As $A W_{M}^{r}(E, F)$ has an order continuous norm, $\left\|T_{\alpha}\right\|=\|f\| \| y_{\alpha}$ $\| \longrightarrow 0$. As a result, $\left\|y_{\alpha}\right\| \longrightarrow 0$.

The condition that $F$ having an order continuous norm is not sufficient for $A W_{M}^{r}(E, F)$ to have an order continuous regular norm. We demonstrate this fact in the following example.

Example 10. We consider $A W_{M}^{r}\left(c_{0}, c_{0}\right)$ and note that $c_{0}$ has an order continuous norm. We claim that $A W_{M}^{r}\left(c_{0}, c_{0}\right)$ does not have an order continuous regular norm. Suppose on the contrary it has. Let $I: c_{0} \longrightarrow c_{0}$ be the identity operator. Then, $I$ is a positive almost M-weakly compact operator ([12], p. 143). The order interval $[0, I]$ is the same in $A W_{M}^{r}($ $E, F)$ and in $\mathscr{L}^{r}(E, F)$ (by Theorem 2). So the norm is order continuous on $[0, I]$. By Proposition 3.6.19 of [2], we infer that the identity operator $I$ is $\mathrm{M}$-weakly compact, a contradiction ([12], p. 143).

Below we give some sufficient conditions under which $A W_{M}^{r}(E, F)$ admits an order continuous regular norm.

Proposition 11. Let $E$ and $F$ be Banach lattices. If one of the following conditions holds, then $A W_{M}^{r}(E, F)$ has an order continuous regular norm.

(i) $E^{\prime}$ and $F^{\prime \prime}$ have an order continuous norm.

(ii) $F$ is reflexive.

(iii) $E$ is an AM-space with unit, and F has an order continuous norm.

Proof. Let $T \in A W_{M}^{r}(E, F)_{+}$. We note that in each part ((i)-(iii)) $F$ has an order continuous norm. By Proposition 3.6.19 of [2], it is sufficient to show that $T$ is M-weakly compact. Suppose (i) holds. Since $T$ is almost M-weakly compact, its adjoint $T^{\prime}$ is almost L-weakly compact. By ([12], Corollary 4,) $T^{\prime}$ is L-weakly compact, and hence, $T$ is M-weakly compact. Now, suppose (ii) holds. Again, $T^{\prime}$ is almost L-weakly compact. Since $F^{\prime}$ is reflexive (because $F$ is reflexive), $T^{\prime}$ is L-weakly compact. Consequently, $T$ is $\mathrm{M}$-weakly compact. Finally, suppose (iii) holds. Since $E$ is an AM-space with unit, $T$ is semicompact ([1], p. 339, Ex. 15). As $F$ has an order continuous norm, $T$ is L-weakly compact ([2], Corollary 3.6.14) and hence weakly compact. It follows from Theorem 5.62 of [1] that $T$ is M-weakly compact, and we are done.

We end with a question: "What is a necessary and sufficient condition for the Banach lattice $A W_{M}^{r}(E, F)$ to have an order continuous regular norm?"

\section{Data Availability}

No data were used to support this study.

\section{Conflicts of Interest}

The authors declare that there is no conflict of interests regarding the publication of this paper.

\section{References}

[1] C. D. Aliprantis and O. Burkinshaw, Positive Operators, Springer, Dordrecht, 2006.

[2] P. Meyer-Nieberg, Banach Lattices, Universitext, SpringerVerlag, Berlin, 1991.

[3] F. Ozger, "Characterizations of compact operators on $\ell_{p}$-type fractional sets of sequences," Demonstratio Mathematica, vol. 52, no. 1, pp. 105-115, 2019.

[4] B. P. Duggal and I. H. Kim, "Structure of n-quasi left minvertible and related classes of operators," Demonstratio Mathematica, vol. 53, no. 1, pp. 249-268, 2020.

[5] A. Aral and V. Gupta, "On the analogue of Stancu-Beta operators," Applied Mathematics Letters, vol. 25, no. 1, pp. 67-71, 2012.

[6] V. Gupta and G. Greubel, "Moment estimations of new SzászMirakyan-Durrmeyer operators," Applied Mathematics and Computation, vol. 271, pp. 540-547, 2015.

[7] U. Abel, V. Gupta, and R. Mohapatra, "Local approximation by a variant of Bernstein Durrmeyer operators," Nonlinear Analysis, vol. 68, no. 11, pp. 3372-3381, 2008.

[8] P. Meyer-Nieberg, "Über Klassen schwach kompakter operatoren in Banachverbänden," Mathematische Zeitschrift, vol. 138, no. 2, pp. 145-159, 1974.

[9] E. Bayram and A. W. Wickstead, "Banach lattices of L-weakly and M-weakly compact operators," Archiv der Mathematik, vol. 108, no. 3, pp. 293-299, 2017.

[10] Z. L. Chen and A. W. Wickstead, "L-weakly and M-weakly compact operators," Indagationes Mathematicae, vol. 10, no. 3, pp. 321-336, 1999.

[11] K. Bouras, D. Lhaimer, and M. Moussa, "On the class of almost L-weakly and almost M-weakly compact operators," Positivity, vol. 22, no. 5, pp. 1433-1443, 2018.

[12] A. Elbour, F. Afkir, and M. Sabiri, "Some properties of almost L-weakly and almost M-weakly compact operators," Positivity, vol. 24, no. 1, pp. 141-149, 2020.

[13] F. Afkir, K. Bouras, A. Elbour, and S. El Filali, "Weak compactness of almost L-weakly and almost M-weakly compact operators," Quaestiones Mathematicae, pp. 1-10, 2020.

[14] J. A. Sanchez, Operators on Banach Lattices (Spanish), [Ph.D. thesis], Complutense University, Madrid, 1985.

[15] W. Wnuk, "Banach lattices with the weak Dunford-Pettis property," Atti del Seminario Matematico e Fisico dell'Università di Modena, vol. 42, no. 1, pp. 227-236, 1994.

[16] B. Aqzzouz and A. Elbour, "Some characterizations of almost Dunford-Pettis operators and applications," Positivity, vol. 15, no. 3, pp. 369-380, 2011.

[17] Z. L. Chen and A. W. Wickstead, "The order properties of rcompact operators on Banach lattices," Acta Mathematica Sinica, English Series, vol. 23, no. 3, pp. 457-466, 2007. 